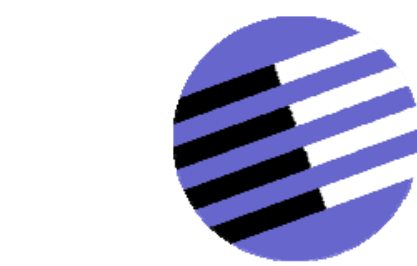

GOVERNANCE AND THE EFFICIENCY

OF ECONOMIC SYSTEMS

GESY

\begin{tabular}{|c|}
\hline Discussion Paper No. 144 \\
When queueing is better \\
than push and shove \\
Alex Gershkov* \\
Paul Schweinzer** \\
\end{tabular}

June 2006

*Alex Gershkov, University of Bonn, Department of Economics, Economic Theory II, Lennéstrasse 37, 53113 Bonn, Germany. Tel: +49 228737993, Fax: +49 228737940, alex.gershkov@uni-bonn.de

**Paul Schweinzer, Department of Economics, University of Bonn Lennéstraße 37, 53113 Bonn, Germany paul.schweinzer@uni-bonn.de

Financial support from the Deutsche Forschungsgemeinschaft through SFB/TR 15 is gratefully acknowledged. 


\title{
When queueing is better than push and shove*
}

\author{
Alex Gershkov'and Paul Schweinzer \\ Department of Economics, University of Bonn \\ Lennéstraße 37, 53113 Bonn, Germany
}

\begin{abstract}
We address the scheduling problem of reordering an existing queue into its efficient order through trade. To that end, we consider individually rational and balanced budget direct and indirect mechanisms. We show that this class of mechanisms allows us to form efficient queues provided that existing property rights for the service are small enough to enable trade between the agents. In particular, we show on the one hand that no queue under a fully deterministic service schedule such as first-come, first-serve can be dissolved efficiently and meet our requirements. If, on the other hand, the alternative is service anarchy (ie. a random queue), every existing queue can be transformed into an efficient order.

(JEL C72, D44, D82. Keywords: Scheduling, Queueing, Mechanism design.)
\end{abstract}

\section{Introduction}

We analyse the problem of organising efficient sequential access of a set of agents to some service. All agents value the service equally but have a privately known waiting cost. We assume that there is an inefficient waiting queue in place upon which we want to improve. Moreover, efficient access is to be organised among the agents themselves, without payments from or to

*Thanks for helpful discussions to Benny Moldovanu, Thomas Gall, Aner Sela, Timofiy Mylovanov, and Motty Perry. Financial support from the German Science Foundation through SFB/TR 15 is gratefully acknowledged.

†Corresponding author: tel +49 22873 7993, fax +49 22873 7940, Alex. Gershkov@uni-bonn. de. 
outsiders. Hence the class of mechanisms we are concerned with is that of the individually rational and balanced budget mechanisms resulting in an efficient allocation. For fully deterministic queues where agents are issued with non-probabilistic slot tickets, it is impossible to achieve an efficient order using a mechanism from the above class. If, however, agents initially face service anarchy, then they can mutually agree to implement the efficient order.

Economic applications of our model include the dynamic assignment of airport landing and takeoff slots, the scheduling of trains through route bottlenecks and ships' servicing at sea ports, or any "control of vehicular traffic congestion" (Naor (1969)). Similarly, the joint scheduling of jobs by different units on a corporate shop floor or the sequential access to research facilities such as supercomputers, radio telescopes or laboratories fits our model well. Leaving ethical considerations aside, a particularly interesting example arises in the waiting system of the British National Health Service (NHS). There, patients for certain procedures (such as nose operations, for example) are put on a waiting list with the ranking being based on their doctors' diagnoses. As a result, patients with the same diagnosis are treated firstcome, first-serve but may have differing and privately known waiting costs. 'Private' patients often use the same facilities, doctors and staff, but are not subject to the same schedule. They are typically treated without significant waiting and their payments are made to the service provider. If trade between queue-positions in a single queue is possible, the payments made by these private patients accrue to the other patients whose wait is prolonged through the speedier servicing of the private patients. Thus the difference to our mechanism is in who gets the money-the service provider or the other patients through our balanced budget requirement. Individual rationality of the mechanism is ensured through the universality principle of the NHS: everyone is entitled to its services and may or may not accept the offered payments for switching positions.

Another example arises with the potential short-term dynamic trade of airport landing and takeoff slots. ${ }^{1}$ A short-term slot trading mechanism—for, say, one hour of takeoff or landing activity in advance-is a scheduling problem since the set of arriving and departing airplanes are known for the period considered. Ball, Donohue, and Hoffman (2006) argue for a near-real-

\footnotetext{
${ }^{1}$ The 2000 AIR-21 Bill prescribes the total deregulation of slot controls at the following US High Density Rule airports for 2007: New York John F Kennedy and LaGuardia, Chicago O'Hare and Washington Reagan National. By that time, dynamic trading of slots between airlines will be possible.
} 
time market that allows for the trading of slots: "A key property of these slot-trading markets is that each airline is potentially both a buyer and seller. In fact, the natural extension of the current exchange system suggests simply adding the possibility of side payments to the current trades." Vindicating our balanced budget condition, they point out that the authorities running airports are "almost always public agencies [and] typically restricted in that their charges for services can only achieve cost recovery."

We view our contribution as mapping two important results into the sphere of queuing. Myerson and Satterthwaite (1983), on the one hand, show the inexistence of efficient and individually rational trading mechanisms for a wide class of incomplete information problems with asymmetric ownership distributions. On the other hand, Cramton, Gibbons, and Klemperer (1987) derive the contrary result that there can be efficient trade among a group of agents provided that initial ownership is equally distributed. We show that the analogue of the former is any deterministic or, in particular, the first-come, first-serve (fcfs) schedule which cannot be efficiently rescheduled and the analogue of latter is the random schedule which can be rescheduled efficiently. Actually, the problem of efficiently reorganising a two player deterministic queue is equivalent to the Myerson-Satterthwaite environment of efficient trade under incomplete information.

Two widely used service schedules are the first-come, first-serve procedure and the random schedule. Since both these procedures are inefficient, we analyse whether there exists another game which implements the efficient allocation and improves the utilities of all players in the queue without making a budget deficit. While we show that such a game indeed exists for the random scheduling, it does not exist for the fcfs scheduling or any other deterministic rule. By a deterministic rule we mean a scheduling rule where an agent is served with probability one at a particular slot. Throughout the paper we use the fcfs schedule as representative for any such deterministic rule.

\section{Related literature}

Most of the existing literature analysing scheduling problems employing Vickrey-Clarke-Groves (VCG) mechanisms ignores individual rationality. The exception is Mitra (2001) who assumes 
that individual rationality is with respect to not getting service at all. In contrast, we show that efficient rescheduling is possible when the alternative is the random queue, ie. when the probability of being served at any slot is equal among agents. We also show that rescheduling is impossible when the alternative is any deterministic rule, as for instance fcfs. Individual rationality with respect to some existing mechanism ensures that no agent is made worse off through moving to the new mechanism.

Previous work on scheduling problems based on the VCG mechanism starts with Dolan (1978). Suijs (1996) assumes linear cost (as we do) and shows that a VCG mechanism implements the efficient order in dominant strategies and balances the budget. Strandenes and Wolfstetter (2005) generalise over Dolan's equal service time and linear cost assumptions. Mitra (2002) shows that linear cost functions are the only cost functions which can lead to an efficient allocation in dominant strategies, if budget balancedness is required. Hain and Mitra (2004) allow for processing time to be private information. They identify the class of mechanisms which lead to efficient allocation and budget balancedness in ex-post equilibrium. The aforementioned analyse VCG mechanisms but do not impose individual rationality with respect to an existing mechanism. Krishna and Perry (1997) analyse a general problem allowing for a mechanism which has an equilibrium featuring an efficient allocation while balancing its budget and being individually rational. An application of their results to our setting, however, is not a simple task. This is not meant to diminish their contribution as their environment and mechanism are much more general than ours.

The queueing literature studies the aspect of our problem that arriving customers can gain priority over others through a single one-off payment to the service provider under the heading of 'priority pricing.' Hassin and Haviv (2002) survey the recent queueing literature including models where the queueing agents offer payments to the service provider. Afèche and Mendelson (2004) analyse queues where the delay cost depends on the service valuation and use auctions to allocate priority. They introduce a multiplicative structure linking delay costs with valuations over the typically additive formulation in the literature following Naor (1969). Kittsteiner and Moldovanu (2005) study priority auctions allowing for private information on processing time. Mitra (2001) is a mechanism design approach aiming at the identification of cost functions for which queues can be efficiently reorganised in dominant strategies 
while balancing the budget. He further derives a subset of individually rational cost functions where non-participation means obtaining no service at all. Recent cooperative approaches to queueing where the assigned positions and payments are based on the Shapley value include Maniquet (2004) and Mishra and Rangarajan (2005). The equally cooperative literature on sequencing games studies the problem of sharing the cost gains in moving from an initially given queue to some optimal ordering. It was surveyed recently by Curiel, Hamers, and Klijn (2002) and focuses on the existence and properties of the cooperative games' core.

We can alternatively take the point of view of matching theory. We may simply drop our interpretation of the private information as waiting cost and the queue as a waiting device and think about the queue as a general ranking of the type with our cost function discriminating between the assigned objects. ${ }^{2}$ From this point of view, our game is an instance of the assignment game with transferable utility due to Shapley and Shubik (1972) under incomplete information with side payments within the set of agents. Our problem thus resembles the multi-item auctions problem solved in dominant strategies by Demange, Gale, and Sotomayor (1986) with the additional constraint of balancing the budget for goods which can be ranked on a single dimension. More specifically, our mechanism can be applied to the problem of the assignment of universally ranked dorm rooms with existing tenants or the allocation of places at universally ranked schools or universities. These or similar problems have been recently analysed in a strictly nontransferable utility setting—and thus contrasting our analysis_-by Abdulkadiroglu and Sönmez (2003) and Sönmez and Ünver (2005) among others. Extending existing results, our mechanism allows for the gains from trade between agents to be realised even when agents have the same ranking over the available objects.

The following section introduces the model. In section 3 we develop the direct revelation game and in section 4 we construct an indirect game implementing the efficient queue. All proofs and details are contained in the appendix.

${ }^{2}$ Our particular specification of the linear cost, unit processing time and the 'ideal' object's valuation need not fit
well with other interpretations than the above quoted school assignment and dormitory allocation examples. 


\section{The model}

There is a set $\mathcal{N}$ of $n>1$ players willing to get some specific service valued at $V$. Although the service is valuable, every player incurs a cost of waiting to get the service. More precisely, for $i \in \mathcal{N}$, we assume that player $i^{\prime}$ s utility from getting the service at the $k^{t h}$ period is $V-k \theta_{i}-p$ where $p$ is a monetary payment by agent $i$ and $\theta_{i}$ is waiting cost per one unit of time. ${ }^{3}$ The server can serve only one player at each point in time. Waiting cost $\theta_{i} \in \Theta_{i}=[0,1]$ is private information and independently distributed with density $f$ and distribution function $F$. Finally, we assume that processing time of any player is the same and normalised to 1 period.

The mechanism designer wishes to implement the efficient order of service, which coincides with a decreasing ranking of the waiting cost. This maximises the aggregated expected utility of the players. By the revelation principle we may restrict attention to direct revelation mechanisms, where the players have to reveal only their private information to the designer. Denote by $\Theta=[0,1]^{n}$ the type space and by $\theta$, any element of $\Theta$. The mechanism $M$ has to specify two things: The payment that each player should pay and the (possibly stochastic) order of getting the service. Therefore a direct revelation mechanism is a vector of payments $p^{M}=\left\langle p_{i}^{M}\right\rangle_{i=1}^{n}$ and the order $\sigma^{M}=\left\langle\sigma_{i j}^{M}\right\rangle_{i, j=1}^{n}$, where

$$
p_{i}^{M}: \Theta \rightarrow \mathbb{R}
$$

is bidder $i$ 's payment and for $1 \leq i, j \leq k$

$$
\sigma_{i j}^{M}(\theta): \Theta \rightarrow[0,1]
$$

specifies the probability that agent $i$ is served at the $j^{t h}$ period. Consequently we have $\sum_{i} \sigma_{i j}^{M}=$ 1 for each $j$ and $\sum_{j} \sigma_{i j}^{M}=1$ for each $i$. Therefore, expected utility of the player $i$ who observes signal $\theta_{i}$ (while the rest of the players observe signals $\theta_{-i}$ ), if all players report their observed signals correctly is

$$
U_{i}\left(\theta_{i}\right)=V-\sum_{k=1}^{n} \sigma_{i k}^{M}(\theta) k \theta_{i}-p_{i}^{M}(\theta)
$$

where $\theta=\left(\theta_{i}, \theta_{-i}\right)$. Denote by $W_{i}^{M}\left(\theta_{i}\right)$ and $P_{i}^{M}\left(\theta_{i}\right)$ the expected waiting time and the payment

\footnotetext{
${ }^{3}$ As customary in the literature, we do not consider discounting of payments.
} 
by player $i$ if $\theta_{i}$ is the reported delay cost. That is

$$
P_{i}^{M}\left(\theta_{i}\right)=\mathbb{E}_{\theta_{-i}} p_{i}^{M}(\theta), \quad W_{i}^{M}\left(\theta_{i}\right)=\sum_{k=1}^{n} k \mathbb{E}_{\theta_{-i}} \sigma_{i k}^{M}(\theta) .
$$

The mechanism $M$ is incentive compatible if, for any $i$ and any $\theta_{i}, \hat{\theta}_{i} \in[0,1]$, it is true that

$$
-W_{i}^{M}\left(\theta_{i}\right) \theta_{i}-P_{i}^{M}\left(\theta_{i}\right) \geq-W_{i}^{M}\left(\hat{\theta}_{i}\right) \theta_{i}-P_{i}^{M}\left(\hat{\theta}_{i}\right) .
$$

Three possible service schedules are of interest to us: a stochastic order, a deterministic schedule determined through something other than the private information and the efficient order.

1. Random order. In this discipline each player can be at any position with equal probability. That is

$$
\sigma_{i k}^{\mathrm{ran}}(\theta)=\frac{1}{n} \text { for any } i, k, \theta \text {. }
$$

2. First-come, first-serve order. In this case, each player is served according to deterministic order based on some exogenous parameter (unrelated to the waiting costs), like the arrival time. That is, for any player $i$ there exists a unique position $l$ such that

$$
\sigma_{i k}^{\mathrm{fffs}}(\theta)=\left\{\begin{array}{ccc}
1 & \text { if } & l=k \\
0 & & \text { otherwise }
\end{array}\right.
$$

3. Efficient order. In this case, players get the service based on decreasing waiting cost, ie.

$$
\sigma_{i k}^{\text {ef }}(\theta)=\left\{\begin{array}{ccc}
1 & \text { if } & \left|\left\{j: \theta_{j}>\theta_{i}\right\}\right|=k \text { and }\left|\left\{j \neq i: \theta_{j}=\theta_{i}\right\}\right|=0 \\
\frac{1}{m} & \text { if } & \left|\left\{j: \theta_{j}>\theta_{i}\right\}\right|=l \text { and }\left|\left\{j \neq i: \theta_{j}=\theta_{i}\right\}\right|=m \neq 0, \text { where } l+m \geq k>l \\
0 & \text { otherwise }
\end{array}\right.
$$

where $|S|$ is the number of elements in set $S$.

In the following we deal with the question of which kind of schedule can be improved upon in the mutual interest. Hence we analyse the question whether there exists a game that induces the efficient allocation, provides all types of all players with expected utilities higher then the 
one obtained in the random or fcfs order while balancing the budget ex post. ${ }^{4}$

\section{Direct mechanisms}

The first lemma specifies necessary and sufficient conditions for the mechanism to be incentive compatible. In particular, it says that in any incentive compatible mechanism, increasing the delay cost should lead to earlier service and higher payment of that player. This is similar to a standard result in auction theory (Myerson (1981), among others) that says that in any incentive compatible mechanism, the probability to get the object increases with a player's valuation. Since the proofs for lemmata 1-4 are standard, they are omitted. ${ }^{5}$

Lemma 1. The mechanism $M=\left\langle p^{M}, \sigma^{M}\right\rangle$ is incentive compatible iff for every $i \in N$ and all $\bar{\theta}, \underline{\theta} \in$ $[0,1], W_{i}^{M}$ is decreasing and

$$
P_{i}^{M}(\underline{\theta})-P_{i}^{M}(\bar{\theta})=\int_{\underline{\theta}}^{\bar{\theta}} s d W_{i}^{M}(s) .
$$

The players prefer to adopt any new mechanism if it provides them with higher utility then the original mechanism. Hence we check whether our proposed mechanism is individually rational when the outside option is either the random scheduling or the first-come, first-serve order. The next lemma specifies the type of each player who gains least among all possible types of that player by moving to our mechanism $\left\langle p^{M}, \sigma^{M}\right\rangle$. More precisely, it says that the net utility is minimised for the type of player who on average stays at the same position.

Lemma 2. Given an incentive compatible mechanism $M=\left\langle p^{M}, \sigma^{M}\right\rangle$, player $i$ 's net utility with respect to mechanism $Z$ is minimised at

$$
\theta_{i}^{*}(Z)=\frac{1}{2}\left[\inf \Theta_{i}^{*}(Z)+\sup \Theta_{i}^{*}(Z)\right] \in[0,1]
$$

where $\Theta_{i}^{*}(Z)=\left\{\theta_{i} \mid W_{i}\left(\theta_{j}\right)<W_{i}^{Z}\left(\theta_{i}\right) \forall \theta_{j}<\theta_{i} ; W_{i}\left(\theta_{k}\right)>W_{i}^{Z}\left(\theta_{i}\right) \forall \theta_{k}>\theta_{i}\right\}$ and $Z \in\{$ ran, fcfs $\}$.

In the next lemma we derive a condition for any incentive compatible mechanism to be individually rational.

\footnotetext{
${ }^{4}$ It is easy to relax our balanced budget condition to allow for a surplus if that should be desired.

${ }^{5}$ For complete proofs of very similar statements see Cramton, Gibbons, and Klemperer (1987).
} 
Lemma 3. An incentive compatible mechanism $M=\left\langle p^{M}, \sigma^{M}\right\rangle$ is individually rational with respect to mechanism $Z \in\{$ ran, fcfs $\}$ iff, for all $i \in N$ and $\theta_{i}^{*}(Z)$ as defined in the previous lemma,

$$
P_{i}^{M}\left(\theta_{i}^{*}(Z)\right) \leq 0
$$

The mechanism satisfies budget balance if $\sum_{i=1}^{n} p_{i}^{M}(\theta)=0$. The next lemma specifies a condition for the budget to balance in the mechanism that satisfies incentive compatibility and individual rationality.

Lemma 4. For any expected waiting time $W_{i}^{M}\left(\theta_{i}\right)$ which is decreasing for all $i \in N$, there exists a transfer function $p^{M}$ such that $\left\langle p^{M}, \sigma^{M}\right\rangle$ is incentive compatible, individually rational with respect to mechanism $Z \in\{$ ran, $f c f s\}$ and budget balanced iff, for $\theta_{i}^{*}(Z)$ defined in lemma 2

$$
\sum_{i=1}^{n}\left[\int_{0}^{\theta_{i}^{*}(Z)} s F(s) d W_{i}^{M}(s)-\int_{\theta_{i}^{*}(Z)}^{1} s(1-F(s)) d W_{i}^{M}(s)\right] \geq 0 .
$$

Now we start the analysis of the properties of the efficient schedule.

Lemma 5. In the efficient schedule, type $\theta_{i}$ 's expected waiting time is

$$
W_{i}^{e f}\left(\theta_{i}\right)=n+(1-n) F\left(\theta_{i}\right)
$$

In the following we refer to the efficient queuing schedule as implementable with respect to any other discipline $Z$, if and only if there exists a mechanism $\left\langle p^{M}, \sigma^{\text {ef }}\right\rangle$ which is incentive compatible, individually rational (with respect to $Z$ ) and budget balanced. The next theorem follows from the last lemma and lemma 4.

Theorem 1. Efficient scheduling is implementable with respect to schedule $Z \in\{$ ran, $f c f s\}$ iff

$$
\begin{gathered}
\sum_{i=1}^{n}\left[\int_{0}^{\theta_{i}^{*}(Z)} s F(s) f(s) d s-\int_{\theta_{i}^{*}(Z)}^{1} s(1-F(s)) f(s) d s\right] \leq 0 \\
\text { where } \theta_{i}^{*}(Z)= \begin{cases}F^{-1}\left(\frac{n-k}{n-1}\right) & \text { if } Z=f c f s \\
F^{-1}(1 / 2) & \text { if } Z=\text { ran }\end{cases}
\end{gathered}
$$

and $k$ is the position of player $i$ in the fcfs schedule. 
Notice that the worst possible type in fcfs depends on the position of the player in the initial order. The next proposition shows that for an initially random schedule, it is always possible to reschedule efficiently.

Proposition 2. For any distribution of types $F$, the efficient scheduling is implementable with respect to the random order.

As we show in the following proposition, the opposite holds if the initial schedule is fcfs.

Proposition 3. For any distribution of types $F$, the efficient scheduling is not implementable with respect to first-come, first-serve order.

It is the existing property rights in a service slot which explain the difference between the different outside option mechanisms. The key difference between the random and fcfs initial schedules is that the fcfs order gives players full possession over their time of service (with probability one) while the random order only issues a probabilistic ticket. This concentration of property rights on a single service ticket which comes with the fcfs schedule makes it impossible to efficiently reschedule the queue. The reason is that the agent who is to be served first in the initial schedule knows that he will not 'buy forward' (ie. get earlier service) for sure and thus will not exchange his slot with a marginally higher type behind him for a merely marginal payment.

As follows from the previous results, the insertion of some uncertainty into a deterministic queue (thus making it stochastic) makes efficient rescheduling possible. For instance, consider a lottery which results with probability $p$ in the random queue and probability $1-p$ the fcfs queue. Let this lottery be executed if at least one player disagrees in participating in the efficient mechanism. Since the worst-off type in the lottery is continuous in $p$, for $p$ sufficiently high, there exists an equilibrium where the efficient allocation is implemented.

\section{An efficient indirect mechanism}

As an illustration of our prior results, we now analyse an auction game that implements the efficient schedule. The auction's basic structure is given by the following properties: 
- each player $i$ offers some payment for being served in each position of the queue, ie. each player offers an $n$-vector of bids,

- we assign queue positions $s=1, \ldots, n$ in increasing order; the highest bidder for position $s$ gets this position and pays the own bid for this slot; ${ }^{6}$ the assigned bidder's bids are removed from subsequent slot-allocations,

- every slot's payment is shared in equal amounts by all other players.

Notice that an agent's bid for the $k^{\text {th }}$ slot is relevant only if the bidder did not secure service at any previous slot. Thus given that an agent is still unassigned, he ignores all previous proceedings when deciding on his $k^{\text {th }}$ bid. Therefore, if all bidders' bidding function is given by the increasing function $\beta^{k}\left(\theta_{j}\right)$, then agent $i$ submits the bid $b$ for the $k^{\text {th }}$ slot which maximises

$$
\begin{aligned}
\Pi_{i}^{k}\left(b^{k}\right) & =\operatorname{pr}\left(b>\max _{j \in S}\left\{\beta^{k}\left(\theta_{j}\right)\right\}\right) \mathbb{E}\left[-b-k \theta_{i}+L^{W} \mid b>\max _{j \in S}\left\{\beta^{k}\left(\theta_{j}\right)\right\}\right]+ \\
\operatorname{pr}(b & \left.<\max _{j \in S}\left\{\beta^{k}\left(\theta_{j}\right)\right\}\right) \mathbb{E}\left[\frac{\max \left\{\beta^{k}\left(\theta_{j}\right)\right\}}{n-1}+L^{L} \mid b<\max _{j \in S}\left\{\beta^{k}\left(\theta_{j}\right)\right\}\right]
\end{aligned}
$$

where

$$
L^{W}:=\sum_{l>k} \frac{\max \left\{\tilde{\beta}^{l}\left(\theta_{j}\right)\right\}}{n-1}, \quad L^{L}:=\Pi_{i}^{k+1}\left(b^{k+1}\right)
$$

and $\tilde{\beta}^{l}\left(\theta_{j}\right)$ is the winning bid for slot $l . S$ is the set of the $n-k$ opponents with the lowest types among $n-1$ players, other than $i$. Notice that the above $\Pi_{i}^{k}(\cdot)$ is not agent $i^{\prime}$ s utility. However, if we want to write agent $i$ 's utility as a function only of bids for slot $k$, we obtain an expression like $A+B \Pi_{i}^{k}(\cdot)$, where $A$ and $B$ only depend on the bids for the slots previous to $k$.

Proposition 4. An equilibrium bidding function of the indirect game described above is increasing in the agent's type and is given by

$$
\beta^{k}\left(\theta_{i}\right)=\left(\int_{0}^{\theta_{i}}\left(-k x-L^{L}+L^{W}\right)\left(\int_{0}^{x} \tilde{F}_{k}\left(\theta_{j}\right) d \theta_{j}\right)^{\frac{1}{n-1}} \tilde{F}_{k}(x) d x\right)\left(\int_{0}^{\theta_{i}} \tilde{F}_{k}\left(\theta_{j}\right) d \theta_{j}\right)^{-\frac{n}{n-1}}
$$

\footnotetext{
${ }^{6}$ Ties are broken with equal probability among winners.
} 
for $k=1, \ldots, n-1$, where

$$
\tilde{F}_{k}\left(\theta_{j}\right)=\left(F\left(\theta_{j}\right)\right)^{n-k} \sum_{j=0}^{k-1}\left(\begin{array}{c}
n-k+j-1 \\
n-k-1
\end{array}\right)\left(1-F\left(\theta_{j}\right)\right)^{j}
$$

is the distribution of the $n-k$ highest order statistic among $n-1$ variables.

Note that an agent's payment consists of two parts: He gets the average winning bid of all slots assigned to other players and he pays his own bid for the position at which he is served. Since $\beta^{k}\left(\theta_{i}\right)$ is an increasing function, this mechanism leads to the efficient allocation.

\section{Conclusion}

In this paper we analyse the possibility of rearranging an existing queue into its efficient order through voluntary trade between the queuing agents. Desirable generalisations are over the linear waiting cost and the equal (unit) processing time assumptions. Well known existing results, however, make us pessimistic about the prospects of such generalisations. Another potential generalisation is to extend the model with a stream of stochastically arriving customers and thus turn the scheduling problem into a queueing problem. This will create technical difficulties, but our main conclusion that too strong property rights prevent efficient reordering of the queue will remain in place. Allowing for agents' private information on the time required to complete the service does not make the model more interesting, since this information will be revealed and can be conditioned upon. In case of misrepresenting the service time, fines can be imposed.

\section{Appendix}

Proof of lemma 5. In efficient scheduling, the expected waiting time of type $\theta_{i}$ is given by

$$
W_{i}^{e f}\left(\theta_{i}\right)=\sum_{i=1}^{n} i\left(\begin{array}{c}
n-1 \\
i-1
\end{array}\right)\left(F\left(\theta_{i}\right)\right)^{n-i}\left(1-F\left(\theta_{i}\right)\right)^{i-1}=n+(1-n) F\left(\theta_{i}\right)
$$


where the second equality follows from the expectation of the binomial distribution where the success probability of each trial is $1-F\left(\theta_{i}\right)$, the number of trials is $n-1$, and $i-1$ is the number of successes.

Proof of theorem 1. Inserting (5) into (4) results in (6). The waiting time in the fcfs schedule for position $i$ is given by $i$. Thus the worst-off type $\theta_{i}^{*}(\mathrm{fcfs})$ is

$$
n+(1-n) F\left(\theta_{i}^{*}(\mathrm{fcfs})\right)=i \quad \text { or } \quad F\left(\theta_{i}^{*}(\mathrm{fcfs})\right)=\frac{i-n}{1-n} \quad \text { and } \quad \theta_{i}^{*}(\mathrm{fcfs})=F^{-1}\left(\frac{n-i}{n-1}\right) .
$$

Waiting time in the random queue is

$$
\frac{1}{n} 1+\frac{1}{n} 2+\cdots+\frac{1}{n} n=\frac{1}{n} \sum_{i=1}^{n} i=\frac{1}{n}\left(\frac{n^{2}+n}{2}\right)=\frac{n+1}{2}
$$

and thus the worst-off type $\theta^{*}(\operatorname{ran})$ solves

$$
n+(1-n) F\left(\theta^{*}(\operatorname{ran})\right)=\frac{n+1}{2} \text { or } \quad \theta^{*}(\operatorname{ran})=F^{-1}(1 / 2) .
$$

Proof of proposition 2. We have to show that, for $\theta^{*}=F^{-1}(1 / 2)$,

$$
(1-n)\left[\int_{0}^{\theta^{*}} \theta F(\theta) f(\theta) d \theta-\int_{\theta^{*}}^{1} \theta(1-F(\theta)) f(\theta) d \theta\right] \geq 0
$$

Integration by parts of the first expression between brackets gives

$$
\int_{0}^{\theta^{*}} \theta F(\theta) f(\theta) d \theta=\left.\theta(F(\theta))^{2}\right|_{0} ^{\theta^{*}}-\int_{0}^{\theta^{*}} \theta F(\theta) f(\theta) d \theta-\int_{0}^{\theta^{*}}(F(\theta))^{2} d \theta
$$

and integrating the second expression by parts gives

$$
\int_{\theta^{*}}^{1} \theta(1-F(\theta)) f(\theta) d \theta=-\left.\theta(1-F(\theta))^{2}\right|_{\theta^{*}} ^{1}-\int_{\theta^{*}}^{1} \theta(1-F(\theta)) f(\theta) d \theta+\int_{\theta^{*}}^{1}(1-F(\theta))^{2} d \theta
$$

Therefore we can rewrite the original expression as

$$
(1-n)\left[-\int_{0}^{\theta^{*}} \frac{(F(\theta))^{2}}{2} d \theta-\int_{\theta^{*}}^{1} \frac{(1-F(\theta))^{2}}{2} d \theta\right] \geq 0 .
$$


Proof of proposition 3. Without loss of generality and for notational simplicity we will assume that player $i$ is served in position $i$ in the initial fcfs schedule. We rewrite (6) as claim of non-implementability as

$$
\sum_{i=1}^{n}\left[\int_{0}^{1} \theta F(\theta) f(\theta) d \theta-\int_{\theta_{i}^{*}}^{1} \theta f(\theta) d \theta\right]>0, \quad \text { for } \theta_{i}^{*}=F^{-1}\left(\frac{n-i}{n-1}\right) .
$$

Using (8) on the first term in brackets and integration by parts on the second term gives

$$
-\frac{n}{2}-\frac{n}{2} \int_{0}^{1}(F(\theta))^{2} d \theta+\sum_{i=1}^{n}\left[\int_{\theta_{i}^{*}}^{1} F(\theta) d \theta+\theta_{i}^{*} \frac{n-i}{n-1}\right]>0
$$

which transforms into

$$
\pi(F(\theta)):=\sum_{i=1}^{n}\left[\int_{\theta_{i}^{*}}^{1} F(\theta)-\frac{n-i}{n-1}\right] d \theta-\frac{n}{2} \int_{0}^{1}(F(\theta))^{2} d \theta>0
$$

since

$$
\int_{\theta_{i}^{*}}^{1} \frac{n-i}{n-1} d \theta=\frac{n-i}{n-1}\left(1-\theta^{*}\right) \quad \text { and } \quad \sum_{i=1}^{n} \frac{n-i}{n-1}=\frac{n}{2} .
$$

For any distribution of types $F[\theta]$, we can thus rewrite (6) as the claim that $\pi(F(\theta))>0$. Now define a distribution $F^{*}(\theta)$, which puts all probability mass at the single point $A \in[0,1]$ and thus removes all uncertainty about the agent's type. Below we show that for any distribution $F(\theta)$ that is different from $F^{*}(\theta)$, it is true that

$$
\pi(F(\theta))>\pi\left(F^{*}(\theta)\right), \text { where } F^{*}(\theta)= \begin{cases}0 & \text { if } \theta<A \\ 1 & \text { if } \theta \geq A\end{cases}
$$

Since $\pi\left(F^{*}(\theta)\right)=n(1-A)-(1-A) \sum_{i=1}^{n} \frac{n-i}{n-1}-\frac{n}{2}(1-A)=0$ for any $A \in[0,1]$, this would complete our proof. We show (9) in two steps. In the first step we show that, for any distribution function $F(\theta)$, it is true that

$$
\pi(F(\theta))>\pi(\hat{F}(\theta))
$$

where $\hat{F}(\theta)$ is a distribution function that has no positive measure with positive density and has at most $n-1$ mass points (ie. a discrete distribution). In the second step we show that 
gathering any two mass points from $\hat{F}(\theta)$ into a single mass point must decrease $\pi$.
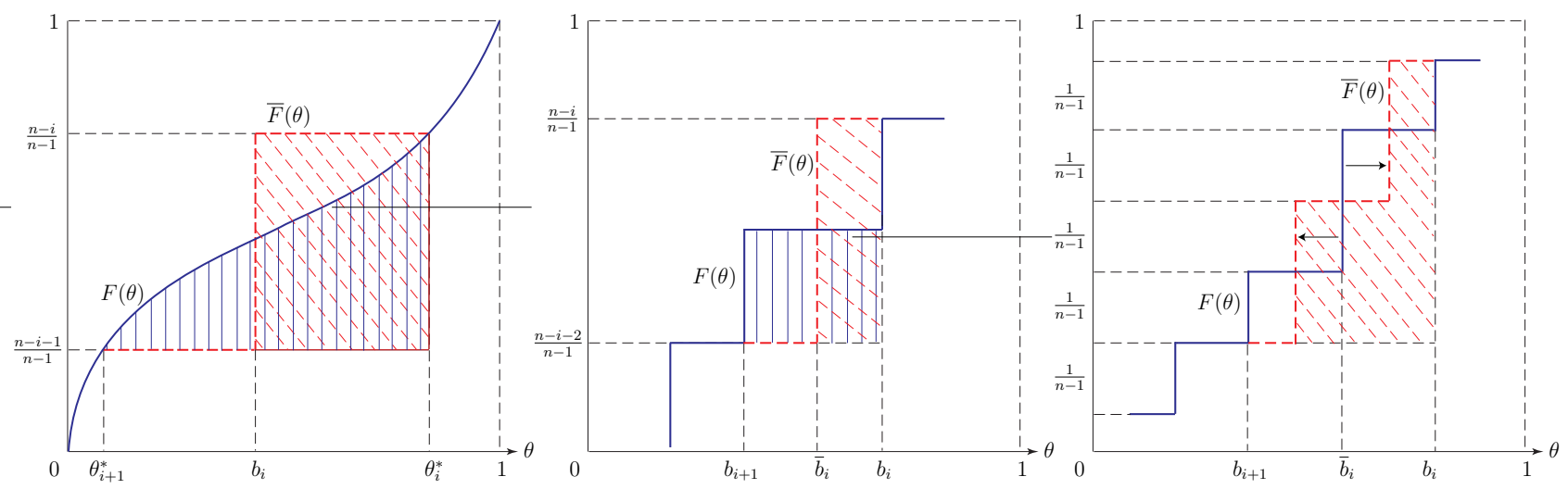

Figure 1: Step 1 (left): The area under the solid $F(\theta)$ is replaced by the equally sized rectangle under the dashed $\bar{F}(\theta)$. Step 2 (centre): Combining two steps of the solid $F(\theta)$ into a single step of equivalent 'virtual' weight. Right: Redistributing a double mass point in $F(\theta)$ into its neighbours.

Step 1. Since in the following we will change the distribution function, denote by $\theta_{i}^{*}(F)$ the worst type of player $i$ if the underlying probability is $F$, which was specified in lemma 2 . In this step we show that if, for some $i, \theta_{i+1}^{*}(F)<\theta_{i}^{*}(F)$ then $\pi(F(\theta))>\pi(\bar{F}(\theta))$ where $\bar{F}(\theta)$ is defined in the following way

$$
\bar{F}(\theta)=\left\{\begin{array}{ccc}
F(\theta) & \text { if } & \theta<\theta_{i+1}^{*}(F) \text { or } \theta \geq \theta_{i}^{*}(F) \\
F\left(\theta_{i+1}^{*}\right) & \text { if } & \theta_{i+1}^{*}(F) \leq \theta<b_{i} \\
F\left(\theta_{i}^{*}\right) & \text { if } & b_{i} \leq \theta<\theta_{i}^{*}(F)
\end{array}\right.
$$

and $b_{i}$ is the solution to

$$
\frac{1}{n-1}\left(F^{-1}\left(\frac{n-i}{n-1}\right)-b_{i}\right)=\int_{F^{-1}\left(\frac{n-i-1}{n-1}\right)}^{F^{-1}\left(\frac{n-i}{n-1}\right)}\left(F(\theta)-\frac{n-i-1}{n-1}\right) d \theta .
$$

Notice that the boundary points $\theta^{*}$ of the new distribution $\bar{F}(\theta)$ coincide: $\theta_{i}^{*}(\bar{F})=\theta_{i+1}^{*}(\bar{F})=$ $b_{i}$. By choice of $b_{i}$ the first term of $\pi(\bar{F}(\theta))$ does not change, while the change in the second term is

$$
\begin{gathered}
\int_{0}^{1} F(\theta)^{2} d \theta-\int_{0}^{1} \bar{F}(\theta)^{2} d \theta=\int_{F^{-1}\left(\frac{n-i-1}{n-1}\right)}^{F^{-1}\left(\frac{n-i}{n-1}\right)} F(\theta)^{2} d \theta- \\
\left(\left(b_{i}-F^{-1}\left(\frac{n-i-1}{n-1}\right)\right)\left(\frac{n-i-1}{n-1}\right)^{2}+\left(F^{-1}\left(\frac{n-i}{n-1}\right)-b_{i}\right)\left(\frac{n-i}{n-1}\right)^{2}\right) .
\end{gathered}
$$


Below we show that $\int_{0}^{1} F(\theta)^{2} d \theta-\int_{0}^{1} \bar{F}(\theta)^{2} d \theta$ is negative. Notice that the second line of the previous expression can be rewritten as

$$
\begin{aligned}
& \left(\frac{n-i-1}{n-1}\right)^{2}\left(F^{-1}\left(\frac{n-i}{n-1}\right)-F^{-1}\left(\frac{n-i-1}{n-1}\right)\right)+\frac{1}{(n-1)^{2}}\left(F^{-1}\left(\frac{n-i}{n-1}\right)-b_{i}\right)+ \\
& \frac{2(n-i-1)}{(n-1)^{2}}\left(F^{-1}\left(\frac{n-i}{n-1}\right)-b_{i}\right)=\left(\frac{n-i-1}{n-1}\right)^{2}\left(F^{-1}\left(\frac{n-i}{n-1}\right)-F^{-1}\left(\frac{n-i-1}{n-1}\right)\right)+ \\
& \frac{2 n-2 i-1}{(n-1)} \int_{F^{-1}\left(\frac{n-i-1}{n-1}\right)}^{F^{-1}\left(\frac{n-i}{n-1}\right)}\left(F(\theta)-\frac{n-i-1}{n-1}\right) d \theta .
\end{aligned}
$$

Therefore, we can rewrite $\int_{0}^{1} F(\theta)^{2} d \theta-\int_{0}^{1} \bar{F}(\theta)^{2} d \theta$ as follows

$$
\begin{aligned}
& \int_{F^{-1}\left(\frac{n-i-1}{n-1}\right)}^{F^{-1}\left(\frac{n-i}{n-1}\right)} F(\theta)\left(F(\theta)-\frac{2 n-2 i-1}{(n-1)}\right) d \theta+ \\
& \left(F^{-1}\left(\frac{n-i}{n-1}\right)-F^{-1}\left(\frac{n-i-1}{n-1}\right)\right)\left(\frac{(n-i-1)(2 n-2 i-1)-(n-i-1)^{2}}{(n-1)^{2}}\right)= \\
& \int_{F^{-1}\left(\frac{n-i-1}{n-1}\right)}^{F^{-1}\left(\frac{n-i}{n-1}\right)} F(\theta)\left(F(\theta)-\frac{2 n-2 i-1}{(n-1)}\right) d \theta+ \\
& \left(F^{-1}\left(\frac{n-i}{n-1}\right)-F^{-1}\left(\frac{n-i-1}{n-1}\right)\right) \frac{(n-i-1)(n-i)}{(n-1)^{2}} .
\end{aligned}
$$

Notice that since

$$
\begin{gathered}
\max _{x \in\left[\frac{n-i-1}{n-1}, \frac{n-i}{n-1}\right]} x \quad\left(x-\frac{2 n-2 i-1}{(n-1)}\right)\left(F^{-1}\left(\frac{n-i}{n-1}\right)-F^{-1}\left(\frac{n-i-1}{n-1}\right)\right) \\
>\quad \int_{F^{-1}\left(\frac{n-i-1}{n-1}\right)}^{F^{-1}\left(\frac{n-i}{n-1}\right)} F(\theta)\left(F(\theta)-\frac{2 n-2 i-1}{(n-1)}\right) d \theta
\end{gathered}
$$

it is sufficient to show that

$$
\begin{gathered}
\max _{x \in\left[\frac{n-i-1}{n-1}, \frac{n-i}{n-1}\right]} x\left(x-\frac{2 n-2 i-1}{(n-1)}\right)\left(F^{-1}\left(\frac{n-i}{n-1}\right)-F^{-1}\left(\frac{n-i-1}{n-1}\right)\right) \\
+\left(F^{-1}\left(\frac{n-i}{n-1}\right)-F^{-1}\left(\frac{n-i-1}{n-1}\right)\right) \frac{(n-i-1)(n-i)}{(n-1)^{2}} \leq 0 .
\end{gathered}
$$

Since for

$$
x=\frac{n-i-1}{n-1} \quad \text { and } \quad x=\frac{n-i}{n-1}
$$


the expression above is zero, we are done. Our argument allows us to restrict attention to distributions which have at most $n-1$ mass points where the probability of any mass point is $k /(n-1)$ where $k$ is natural number.

Step 2. Note that step 1 allows us to restrict attention to discrete distributions with $n-1$ mass points. After step 1 , every mass point has probability $1 /(n-1)$.

Since $\pi(F(\theta))$ is continuous in $b_{i}$ and $1 \geq b_{i-1} \geq b_{i} \geq b_{i+1} \geq 0$, we can conclude that there exist $b_{1}^{*} \geq \ldots \geq b_{n-1}^{*}$ that minimises $\pi(F(\theta))$. To complete the proof, we show that if there is an $i$ such that $b_{i}>b_{i+1}$, then $\pi(F(\theta))>\pi(\bar{F}(\theta))$ where $\bar{F}(\theta)$ is defined as

$$
\bar{F}(\theta)=\left\{\begin{array}{ccc}
F(\theta) & \text { if } & \theta<b_{i+1} \text { or } \theta \geq b_{i} \\
\frac{n-i-2}{n-1} & \text { if } & b_{i+1} \leq \theta<\bar{b}_{i} \\
\frac{n-i}{n-1} & \text { if } & \bar{b}_{i} \leq \theta<b_{i}
\end{array}\right.
$$

and $\bar{b}_{i}$ is given by $\left(\bar{b}_{i}-b_{i+1}\right)(n-i-1)=\left(b_{i}-\bar{b}_{i}\right)(n-i)$ or

$$
\bar{b}_{i}=\frac{b_{i}(n-i)+b_{i+1}(n-i-1)}{2 n-2 i-1} .
$$

Similarly to the first step, this change does not affect first term of $\pi$. Note that

$$
\begin{aligned}
& \int_{0}^{1} F(\theta)^{2} d \theta-\int_{0}^{1} \bar{F}(\theta)^{2} d \theta \\
& =\left(\frac{n-i-1}{n-1}\right)^{2}\left(b_{i}-b_{i+1}\right)-\left(\frac{n-i-2}{n-1}\right)^{2}\left(\bar{b}_{i}-b_{i+1}\right)-\left(\frac{n-i}{n-1}\right)^{2}\left(b_{i}-\bar{b}_{i}\right) \\
& =\left(\frac{n-i-1}{n-1}\right)^{2}\left(b_{i}-b_{i+1}\right)-\left(\frac{n-i-1}{n-1}\right)^{2}\left(\bar{b}_{i}-b_{i+1}\right)-\left(\frac{n-i-1}{n-1}\right)^{2}\left(b_{i}-\bar{b}_{i}\right) \\
& -\frac{1-2(n-i-1)}{(n-1)^{2}}\left(\bar{b}_{i}-b_{i+1}\right)-\frac{1+2(n-i-1)}{(n-1)^{2}}\left(b_{i}-\bar{b}_{i}\right) \\
& =-\frac{1}{(n-1)^{2}}\left(b_{i}-b_{i+1}\right)-\frac{2(n-i-1)}{(n-1)^{2}}\left(b_{i}+b_{i+1}-2 \bar{b}_{i}\right) .
\end{aligned}
$$

Plugging the definition of $\bar{b}_{i}$ into the last expression gives us

$$
-\frac{1}{(n-1)^{2}}\left(b_{i}-b_{i+1}\right)+\frac{2(n-i-1)}{(n-1)^{2}} \frac{b_{i}-b_{i+1}}{2 n-2 i-1}=-\frac{b_{i}-b_{i+1}}{(n-1)^{2}}\left[1-\frac{2(n-i-1)}{2 n-2 i-1}\right]<0
$$

which completes the argument. Notice that after the first application of step 2, the com- 
bined mass point has probability mass of $2 /(n-1)$. In order to be able to apply step 2 again, one can think of this one point as actually consisting of two mass points of equal probability of $1 /(n-1)$ each. Reapplying step 2 to combine these into their respective neighbouring mass points then makes no problems. This is illustrated in the right hand panel of figure 1.

Proof of proposition 4. Agent $i$ chooses $b$ to maximise

$$
\begin{aligned}
& \Pi_{i}^{k}\left(b^{k}\right)= \operatorname{pr}\left(b>\max _{j \in S}\left\{\beta^{k}\left(\theta_{j}\right)\right\}\right) \mathbb{E}\left[-b-k \theta_{i}+L^{W} \mid b>\max _{j \in S}\left\{\beta^{k}\left(\theta_{j}\right)\right\}\right]+ \\
& \operatorname{pr}\left(b<\max _{j \in S}\left\{\beta^{k}\left(\theta_{j}\right)\right\}\right) \mathbb{E}\left[\frac{\max \left\{\beta^{k}\left(\theta_{j}\right)\right\}}{n-1}+L^{L} \mid b<\max _{j \in S}\left\{\beta^{k}\left(\theta_{j}\right)\right\}\right] \\
& \text { where } L^{W}:=\sum_{l>k} \frac{\max \left\{\tilde{\beta}^{l}\left(\theta_{j}\right)\right\}}{n-1}, \text { and } L^{L}:=\Pi_{i}^{k+1}\left(b^{k+1}\right) .
\end{aligned}
$$

$L^{W}$ can be interpreted as the slot $k$ winner's utility from the opponents' payments for the slots auctioned after $k . L^{L}$ is the expected utility a bidder who does not win slot $k$ (or any previous slot) gets from the auctioning of slots after $k$. Since bidding functions are monotonically increasing, we know that $\operatorname{pr}\left(b>\max _{j \in S}\left\{\beta^{k}\left(\theta_{j}\right)\right\}\right)=$

$\tilde{F}_{k}\left(\beta^{k^{-1}}(b)\right):=\operatorname{pr}\left(\theta_{j}<\beta^{k^{-1}}(b) \forall j \in S\right)=\left(F\left(\beta^{k^{-1}}(b)\right)\right)^{n-k} \sum_{j=0}^{k-1}\left(\begin{array}{c}n-k+j-1 \\ n-k-1\end{array}\right)\left(1-F\left(\beta^{k^{-1}}(b)\right)\right)^{j}$.

Using this notation, we can rewrite (10) as

$$
\Pi_{i}^{k}\left(b^{k}\right)=\int_{0}^{\beta^{k^{-1}(b)}}\left(-b-k \theta_{i}+L^{W}\right) \tilde{F}_{k}\left(\theta_{j}\right) d \theta_{j}+\int_{\beta^{k^{-1}(b)}}^{1}\left(\frac{\beta^{k}\left(\theta_{j}\right)}{n-1}+L^{L}\right) \tilde{F}_{k}\left(\theta_{j}\right) d \theta_{j} .
$$

Maximising wrt $b$ gives

$$
\begin{aligned}
\frac{\partial \Pi_{i}^{k}\left(b^{k}\right)}{\partial b}= & -\int_{0}^{\beta^{k^{-1}}(b)} \tilde{F}_{k}\left(\theta_{j}\right) d \theta_{j}+\left(-b-k \theta_{i}+L^{W}\right) \tilde{F}_{k}\left(\beta^{k^{-1}}(b)\right) \frac{1}{\beta^{k^{\prime}}(\hat{\theta})} \\
& -\left(\frac{b}{n-1}+L^{L}\right) \tilde{F}_{k}\left(\beta^{k^{-1}}(b)\right) \frac{1}{\beta^{k^{\prime}}(\hat{\theta})}=0
\end{aligned}
$$


where $\hat{\theta}$ is such that $\beta^{k}(\hat{\theta})=b$. This transforms into the ordinary differential equation

$$
-\int_{0}^{\beta^{k^{-1}}(b)} \tilde{F}\left(\theta_{j}\right) d \theta_{j}-\left(b+k \theta_{i}+\frac{b}{n-1}+L^{L}-L^{W}\right) \frac{\tilde{F}\left(\beta^{k^{-1}}(b)\right)}{\beta^{k^{\prime}}(\hat{\theta})}=0 .
$$

A solution to this is obtained (for the initial condition of $b(0)=0$ ) as

$$
\beta^{k}\left(\theta_{i}\right)=\left(\int_{0}^{\theta_{i}}\left(-k x-L^{L}+L^{W}\right)\left(\int_{0}^{x} \tilde{F}\left(\theta_{j}\right) d \theta_{j}\right)^{\frac{1}{n-1}} \tilde{F}(x) d x\right)\left(\int_{0}^{\theta_{i}} \tilde{F}\left(\theta_{j}\right) d \theta_{j}\right)^{-\frac{n}{n-1}}
$$

which equals (7). Checking the slope of this bidding function gives

$$
\begin{aligned}
\frac{\partial \beta^{k}\left(b^{k}\right)}{\partial \theta_{i}}= & \left(-k \theta_{i}-L^{L}+L^{W}\right)\left(\int_{0}^{\theta_{i}} \tilde{F}\left(\theta_{j}\right) d \theta_{j}\right)^{\frac{1}{n-1}} F\left(\theta_{i}\right) d \theta_{i}\left(\int_{0}^{\theta_{i}} \tilde{F}\left(\theta_{j}\right) d \theta_{j}\right)^{-\frac{n}{n-1}}+ \\
& \left(\tilde{F}\left(\theta_{i}\right)\right)^{-\frac{n}{n-1}} \int_{0}^{\theta_{i}}\left(-k x-L^{L}+L^{W}\right)\left(\int_{0}^{x} \tilde{F}\left(\theta_{j}\right) d \theta_{j}\right)^{\frac{1}{n-1}} \tilde{F}(x) d x
\end{aligned}
$$

where each constituent component is positive, since $L^{W}>L^{L}+k \theta_{i}$.

\section{References}

Abdulkadiroglu, A., And T. Sönmez (2003): "School Choice: A Mechanism Design Approach," American Economic Review, 93(3), 729-47.

AfÈChe, P., And H. Mendelson (2004): “Pricing and Priority Auctions in Queueing Systems with a Genreralised Delay Cost Structure," Management Science, 50(7), 869-82.

Ball, M., G. Donohue, And K. Hoffman (2006): “Auctions for the Safe, Efficient and Equitable Allocation of Airspace System Resources," in Combinatorial Auctions, ed. by P. Cramton, Y. Shoham, and R. Steinberg, chap. 20. MIT Press, Cambridge, MA.

Cramton, P., R. Gibbons, And P. Klemperer (1987): “Dissolving a Partnership Efficiently,” Econometrica, 55, 615-32.

Curiel, I., H. Hamers, and F. Klijn (2002): "Sequencing Games: A Survey," in Chapters in Game Theory: In Honour of Stef Tijs, ed. by P. Borm, and H. Peters, vol. 31 of Theory and Decision Library, pp. 27-50. Kluwer Academic Publishers, Boston. 
Demange, G., D. GAle, And M. Sotomayor (1986): “Multi-item auctions,” Journal of Political Economy, 94(4), 863-72.

Dolan, R. (1978): “Incentive Mechanisms for Priority Queueing Problems," Bell Journal of Economics and Management Science, 9, 421-36.

Hain, R., AND M. Mitra (2004): "Simple Sequencing Problems with Interdependent Costs," Games and Economic Behavior, 48(2), 271-91.

Hassin, R., AND M. HAVIV (2002): To Queue or Not to Queue: Equilibrium Behavior in Queueing Systems, vol. 59 of International Series in Operations Research and Management Science. Kluwer Academic Publishers, Boston.

Kittsteiner, T., And B. Moldovanu (2005): “Priority Auctions and Queue Disciplines that Depend on Processing Time," Management Science, 51(2), 236-48.

KrishnA, V., AND M. PERry (1997): “Efficient Mechanism Design,” Pennsylvania State University, Mimeo.

MANIQUET, F. (2004): “A characterisation of the Shapley Value in Queueing Problems," Journal of Economic Theory, 109(1), 90-103.

Mishra, D., AND B. RAngarajAN (2005): “Cost Sharing in a Job Scheduling Problem," Université Catholique de Louvain, CORE Discussion Paper, 2005/53.

MitrA, M. (2001): “Mechanism Design in Queueing Problems," Economic Theory, 17(2), 277305.

(2002): "Achieving the First Best in Sequencing Problems," Review of Economic Design, 7(1), 75-91.

Myerson, R. B. (1981): “Optimal Auction Design,” Mathematics of Operations Research, 6(1), $58-73$.

Myerson, R. B., And M. A. SAtterthwaite (1983): “Efficient Mechanisms for Bilateral Trading," Journal of Economic Theory, 29(2), 265-281. 
NAOR, P. (1969): “On the Regulation of Queue Size by Levying Tolls," Econometrica, 37(1), $15-24$.

Shapley, L., AND M. SHubiK (1972): “The Assignment Game I: The Core," International Journal of Game Theory, 1(2), 111-30.

SÖNMEZ, T., AND U. ÜNVER (2005): “House Allocation with Existing Tenants: An Equivalence," Games and Economic Behavior, 52(1), 153-85.

Strandenes, S. P., and E. Wolfstetter (2005): “Efficient (Re-)Scheduling: An Auction Approach," Economic Letters, 89(2), 187-92.

Suijs, J. (1996): “On Incentive Compatibility and Budget Balancedness in Public Decision Making," Economic Design, 2(2), 193-209. 